\title{
Hábito de Fumar entre Estudantes de Medicina da Universidade Federal de Pelotas, Brasil: Comparação entre as Prevalências de 1986 e 1991
}

\section{Smoking habits among Students at the Pelotas Medical School: Prevalence in 1986 and 1991}

\section{Ana Maria B. Menezes'; Barnardo L. Horta2; Asandro da Rosa'; Fernando K. Oliveira' \& Marcos Bonnann ${ }^{1}$}

MENEZES, A. M. B.; HORTA, B. L.; ROSA, S.; OLIVEIRA, F. K. E BONNAN, M. Smoking

Habits among Students at the Pelotas Medical School: Prevalence in 1986 and 1991. Cad. Saúde Públ., Rio de Faneiro, 10 (2): 164-170, Apr/fun, 1994.

A cross-sectional survey of smoking habits was carried out among students at the School of Medicine in Pelotas, State of Rio Grande do Sul, Brazil, in 1986 and 1991. The overall participation rate for the originally selected sample was $96 \%$ and $98 \%$ respectively. In 1986, regular smokers comprised $21.6 \%$ of the students and $14.6 \%$ in 1991; former smokers were $7.3 \%$ and $10.9 \%$, respectively.

There was no association between sex, parental smoking, and smoking habits of the students. Students in their last two years of medical training showed an increased prevalence in smoking habits (1991). Respiratory symptoms were associated with smoking habits. It was concluded that the prevalence of smoking is decreasing among students of the School of Medicine in Pelotas.

Key words: Epidemiology; Smoking; Respiratory Symptoms

\section{INTRODUÇÃO}

Reconhece-se, hoje, que a nicotina é tão adictiva quanto a heroína e a cocaína (Pollin, 1982; Pollin \& Revenholt, 1984). Os prejuízos causados à saúde pelo hábito de fumar são amplamente conhecidos (Doll, 1986; IARC, 1986; Lokschin \& Barros, 1983; Ravenholt, 1985; 1990), sendo este considerado pela Organização Mundial de Saúde (OMS) como o maior desafio de Saúde Pública no mundo atual e um dos maiores desafios com que se defronta a Medicina Preventiva de nosso tempo. O controle do vício tabágico fará mais pela saúde do homem e pela sua expectativa de vida do que qualquer outra ação de Medicina Preventiva,

\footnotetext{
${ }^{1}$ Departamento de Clínica Médica da Faculdade de Medicina da Universidade Federal de Pelotas. Avenida Duque de Caxias, 250, Pelotas, RS, 96030-002, Brasil. ${ }^{2}$ Departamento de Medicina Social da Faculdade de Medicina da Universidade Federal de Pelotas. Avenida Duque de Caxias, 250, Pelotas, RS, 96030-002, Brasil.
}

uma vez que, para pessoas que começaram a fumar na adolescência e continuaram a fumar um maço de cigarros por dia, estima-se que a perda média de anos de vida exceda oito anos (Ravenholt, 1990). Apesar disso, este hábito persiste entre estudantes de medicina, médicos e outros profissionais da saúde (Rosemberg \& Perón, 1990), ainda que com uma prevalência menor que a observada na população.

Os médicos desempenham um papel de extrema importância na luta contra o tabagismo, pois o exemplo dos mesmos influenciará o hábito de fumar da população (Sande et al., 1990). Torna-se necessário que as faculdades de medicina informem aos seus estudantes sobre os perigos do tabaco à saúde, como também de sua influência como futuros médicos sobre o tabagismo da população.

Isto levou-os a realizar um estudo para determinar a prevalência do hábito de fumar entre os estudantes de Medicina da Universidade Federal de Pelotas, como também comparar essa prevalência com a verificada em 1986. 
O presente trabalho teve também como objetivos investigar a relação entre hábito de fumar e presença de alguns sintomas respiratórios e verificar a associação entre o hábito de fumar dois filhos e o hábito de fumar dos pais.

\section{MATERIAL E MÉTODOS}

Foi realizado um estudo transversal entre todos os alunos matriculados do primeiro ao quinto ano da Faculdade de Medicina da Universidade Federal de Pelotas. Em 1986 foram entrevistados 426 alunos ( $96 \%$ do total), enquanto em 1991 foram entrevistados 470 alunos ( $98 \%$ do total). Em relação ao questionário utilizado em ambas as pesquisas, algumas diferenças devem ser salientadas: a sintomatologia respiratória em 1986 foi avaliada através da presença de tosse produtiva nos dois últimos anos, enquanto em 1991 foi investigada a presença de tosse seca, tosse produtiva ou chiado, sintomas estes presentes sem resfriado, tendo ocorrido no último ano. Outra diferença foi quanto à forma de aplicação do questionário: em 1991 o questionário foi auto-aplicável, uma mudança que teve como finalidade agilizar o processo de coleta de dados e, com isso, diminuir o percentual de perdas. Em relação à definição de fumantes, em 1986, as pessoas que fumassem pelo menos quatro cigarros por semana durante pelo menos um mês foram considerados fumantes, enquanto em 1991 considerou-se como fumantes aqueles que fumassem um ou mais cigarros por dia, durante pelo menos um mês. Ex-fumantes, em ambos os estudos, foram aque- les que, no período da entrevista, não eram fumantes regulares, mas o haviam sido no passado. Não nos parece que as diferenças acima citadas possam ter influído de maneira significativa em quaisquer dos resultados apresentados. Em relação ao diferente método de aplicação do questionário, achamos pouco provável qualquer tendenciosidade, devido à objetividade das questões e ao fato de a população ser composta por estudantes universitários.

$\mathrm{O}$ teste do qui-quadrado foi utilizado para verificar a existência de associações entre as variáveis.

\section{RESULTADOS}

Houve uma redução na prevalência de fumantes de $21,6 \%$ para $14,9 \%$ entre 1986 e 1991 , às custas de um aumento na prevalência de alunos que nunca fumaram $(71,1 \%$ e $74,3 \% \mathrm{~A})$ e ex-fumantes (7,3\% e 10,9\%), conforme mostra a Figura 1 . A Tabela 1 mostra a prevalência de tabagismo conforme o sexo, enquanto, entre os homens, houve um aumento no percentual de alunos que nunca fumaram de 68,1\%, em 1986, para $76,0 \%$, em 1991 , e uma diminuição no percentual de fumantes de 21,9\%, em 1986, para $15,0 \%$, em 1991. Esta diferença, entretanto, não chegou a ser estatisticamente significativa $(\mathrm{p}=$ 0,08 ). Já entre as mulheres houve um aumento na prevalência de ex-fumantes de 2,6\%, em 1986, para $14,1 \%$, em 1991, e uma diminuição na prevalência de fumantes de $21,2 \%$ para $14,7 \mathrm{a} \%$, e de não-fumantes de $76,3 \%$ para $71,2 \%$, sendo estas diferenças estatisticamente significativas.

FIGURA 1. Comparação entre a Prevalência do Tabagismo: 1986-1991

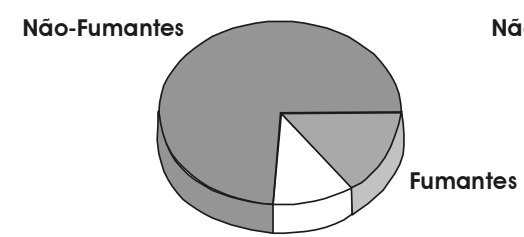

-Fumantes

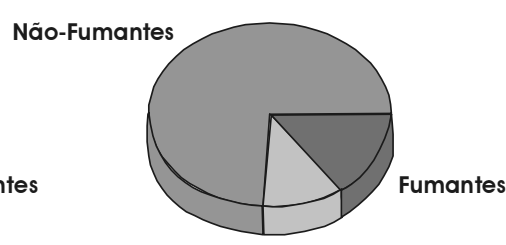

-Fumantes 
TABELA 1. Prevalência do Hábito de Fumar entre os Estudantes de Medicina, Conforme o Sexo e o Ano do Estudo, Pelotas, 1986 e 1991

\begin{tabular}{lcc}
\hline \hline & \multicolumn{2}{c}{ Ano de Estudo } \\
\cline { 2 - 3 } & $1986(\%)$ & $1991 \mathrm{a}(\%)$ \\
\hline Sexo Masculino & 68,1 & 76,0 \\
Nunca fumou & 21,9 & 15,0 \\
Fumante & 10,0 & 9,0 \\
Ex-fumante & & \\
Sexo Feminino & 76,3 & 71,2 \\
Nunca fumou & 21,2 & 14,7 \\
Fumante & 2,6 & 14,1 \\
Ex-fumante & 426 & 470 \\
\hline Total &
\end{tabular}

${ }^{\star} \mathrm{p}<0.001$

Houve uma diminuição, embora não-significativa, no número de cigarros consumidos diariamente pelos fumantes e ex-fumantes, com médias de 12,9, em 1986, e 11,6, em 1991 (Tabela 2).

A Figura 2 apresenta a prevalência de fumantes conforme o ano do curso médico; em 1991 houve uma maior prevalência nos últimos anos do curso médico em relação às séries anteriores, ao contrário do que foi observado em 1986, onde, no último ano, houve uma diminuição na prevalência do tabagismo.

TABELA 2. Consumo Diário de Cigarros entre os Fumantes e Ex-Fumantes, Pelotas, 1986 e 1991

\begin{tabular}{lcc}
\hline \hline & \multicolumn{2}{c}{ Ano de Estudo } \\
\cline { 2 - 3 } Consumo Diário de Cigarros & $1986(\%)$ & $1991 \mathrm{a}(\%)$ \\
\hline $1-9$ & 32,5 & 39,4 \\
$10-19$ & 35,0 & 31,1 \\
$20-39$ & 30,9 & 29,4 \\
40 ou mais & 1,6 & 0,0 \\
\hline Média e & 12,9 & 11,6 \\
Desvio Padrão & $(8,2)$ & $(7,5)$ \\
\hline \hline
\end{tabular}

FIGURA 2. Prevalência do Tabagismo conforme o Ano Cursado

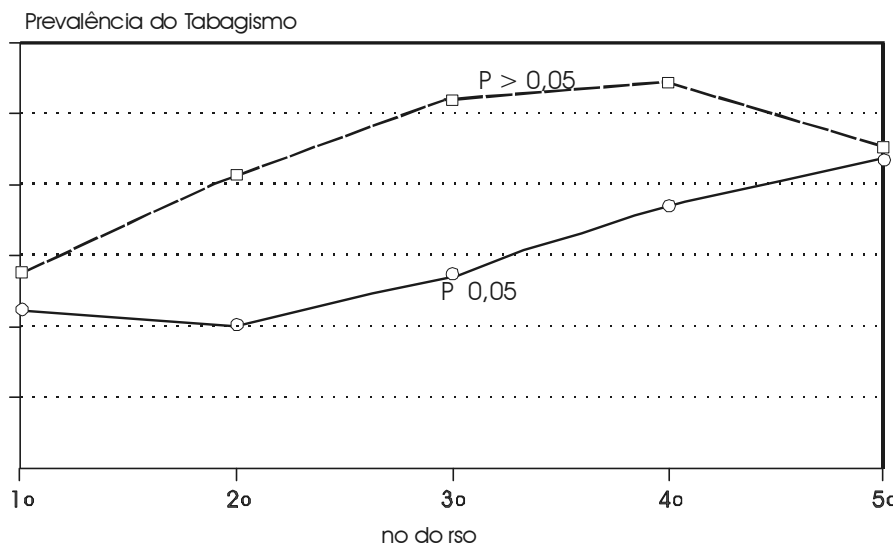


A Tabela 3 mostra que, em relação à idade com que os alunos começaram a fumar, em 1986, a média foi de 17 anos, sendo a moda 15 anos, enquanto em 1991 as mesmas foram de 18 e 17 anos, respectivamente. Tais diferenças não se mostraram estatisticamente significativas.

Em 1991 não foi encontrada uma associação entre o tabagismo dos pais e dos filhos (Tabela 4).

Com relação à associação entre sintomatologia respiratória e o hábito de fumar, em virtude da diferença forma de se estudar esta variável nos dois estudos, conforme discutido acima, serão apresentados aqui apenas os dados de 1991. Como mostra a Figura 3, houve uma associação significativa entre o tabagismo e a freqüência de sintomas respiratórios, tais como tosse seca, tosse produtiva e chiado.

A Figura 4 mostra o hábito de fumar conforme o sexo e ano do curso médico. Em ambos os sexos houve uma diminuição na proporção de não-fumantes a medida que o curso progredia. Esta diminuição foi mais acentuada entre as mulheres, pois no primeiro ano $71,0 \%$ das mesmas e $77,6 \%$ dos homens eram nãofumantes, enquanto no quinto ano $51,7 \%$ das mulheres e $69,0 \%$ dos homens nunca haviam fumado.

TABELA 3. Idade de Início do Fumo, Pelotas, 1986 e 1991

\begin{tabular}{lcc}
\hline \hline & \multicolumn{2}{c}{ Ano de Estudo } \\
\cline { 2 - 3 } Idade de Início & 1986 & 1991 \\
do Tabagismo (anos) & $(\%)$ & $(\%)$ \\
\hline $10-4$ & 12,3 & 13,4 \\
$15-19$ & 72,9 & 65,5 \\
$\geq 20$ & 14,8 & 21,0 \\
\hline Média e & 17,0 & 18,0 \\
Desvio Padrão & $(2,7)$ & $(4,1)$ \\
\hline Moda & 15 & 17 \\
\hline \hline
\end{tabular}

TABELA 4. Relação entre o Hábito de Fumar dos Pais e dos Filhos, Pelotas, 1991

\begin{tabular}{lcccc}
\hline \hline & \multicolumn{3}{c}{ Hábito de Fumar dos Estudantes } & \\
\cline { 2 - 4 } $\begin{array}{l}\text { Hábito de Fumar } \\
\text { dos Pais }\end{array}$ & $\begin{array}{c}\text { Nunca Fumaram } \\
(\%)\end{array}$ & $\begin{array}{c}\text { Fumantes } \\
(\%)\end{array}$ & $\begin{array}{c}\text { Ex-Fumantes } \\
(\%)\end{array}$ & Total \\
\hline Pai & 79,6 & 10,2 & 10,2 & 147 \\
$\quad$ Nunca fumou & 69,9 & 20,8 & 9,5 & 168 \\
$\quad$ Fumante & 74,0 & 13,3 & 12,7 & 150 \\
Ex-fumante & & & & \\
\hline Mãe & 75,2 & 14,6 & 10,1 & 335 \\
Nunca fumou & 67,1 & 20,5 & 12,3 & 73 \\
Fumante & 78,7 & 9,8 & 11,5 & 61 \\
$\quad$ Ex-fumante & 349 & 70 & 50 & 469 \\
\hline Total & & & & \\
\hline \hline
\end{tabular}


FIGURA 3. Sintomas Respiratórios conforme o Tabagismo

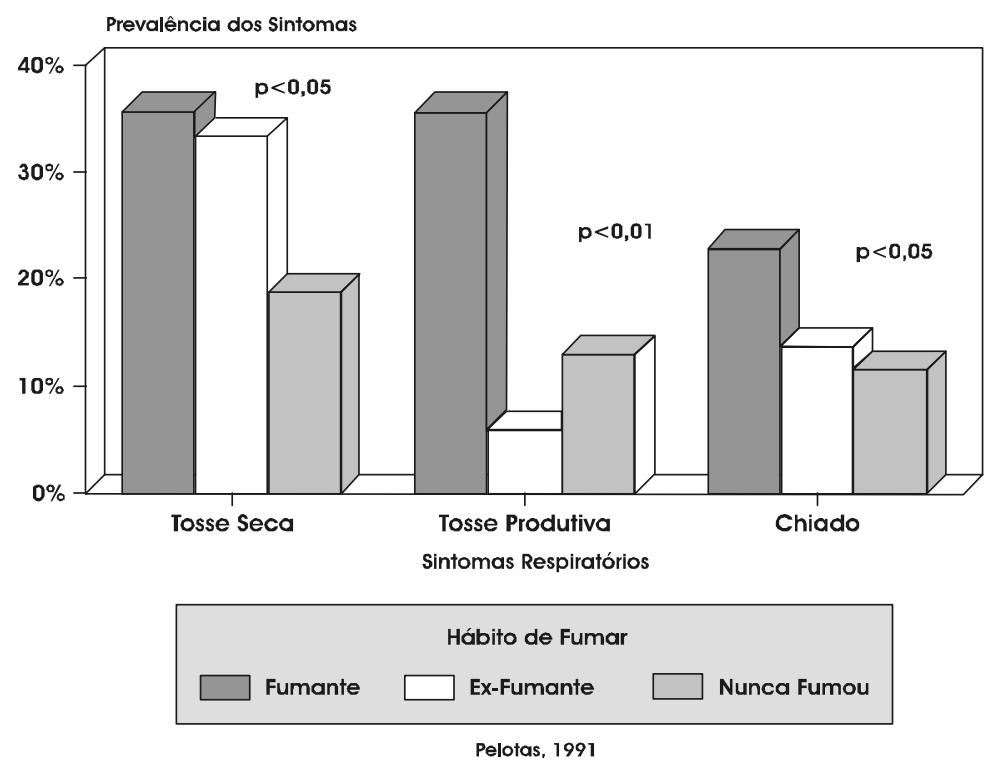

FIGURA 4. Hábito de Fumar conforme o Sexo e o Ano do Curso Médico

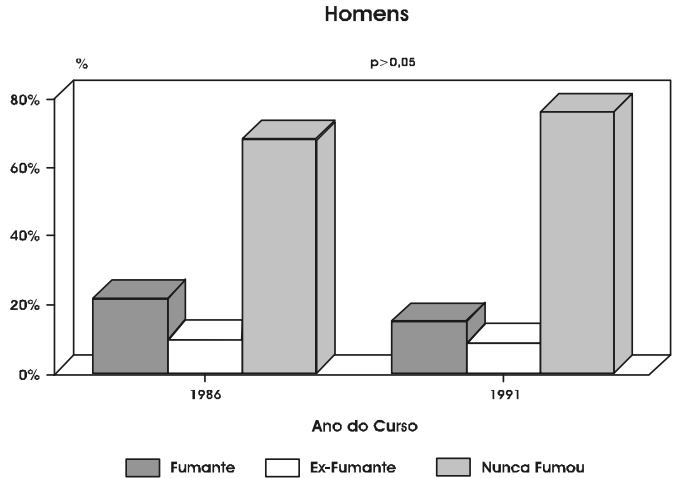

Pelotas, 1991

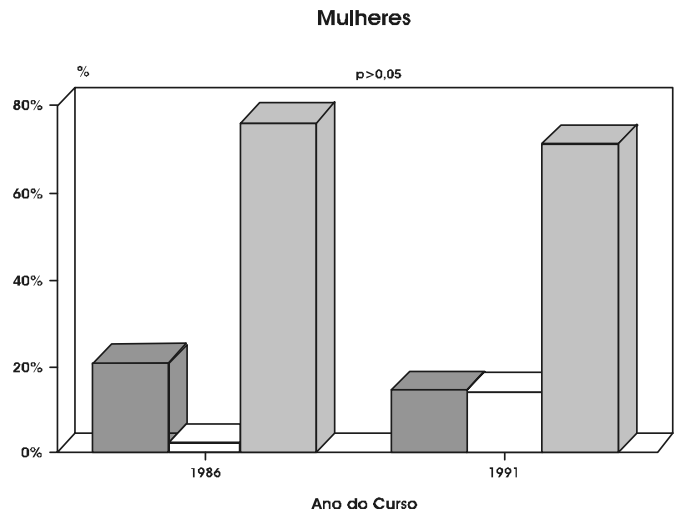

A diminuição estatisticamente significativa na prevalência do tabagismo de 1986 para 1991 (21,6\% para $14,9 \%)$ também foi encontrada em três outras escolas médicas que realizaram estudo semelhante (Rosemberg \& Perón, 1990): Faculdade de Ciências Médicas de Sorocaba, 1979/ 89 (37,8\% para 17,1\%); Faculdade de Medicina 
de Ribeirão Preto, 1980/88 (28,4\% para 11,9\%); e Escola Paulista de Medicina, 1982/89 (21,4\% para $15,6 \%)$. Apesar de estes estudos terem sido realizados com intervalos de tempo diferentes, eles demonstram claramente uma diminuição na prevalência do tabagismo entre estudantes de medicina. Este fenômeno também tem sido observado em estudos populacionais realizados em outros países (Pollin, 1982; Pollin \& Ravenholt, 1984), mas, como não existem estudos sobre a tendência secular ao tabagismo em nosso país, não é possível afirmar se este decréscimo é um efeito isolado entre os jovens que estudam medicina ou o reflexo de uma situação que está ocorrendo em nosso meio.

É importante salientar que, apesar de a população estudada ser constituída de indivíduos jovens e, portanto, fumantes há pouco tempo, encontrou-se uma alta prevalência de sintomas respiratórios entre os fumantes, tais como tosse seca, tosse produtiva ou chiado. Cabe ressaltar, ainda, que sintomas como tosse seca ou chiado poderiam ser decorrentes de patologias como asma brônquica; entretanto, o alto percentual de tosse produtiva leva-nos a pensar principalmente em bronquite crônica, que tem como principal fator de risco o tabagismo (Ravenholt, 1990). Embora a literatura cite que a principal faixa etária da bronquite crônica seja a partir dos 40 anos (Richards \& Baker, 1988), não podemos deixar de levantar a hipótese de que talvez o sintoma acima mencionado se inicie mais precocemente, podendo talvez servir como alerta.

A inexistência de uma associação entre os tabagismo dos pais e o hábito de fumar dos filhos, encontrada tanto em 1986 como em 1991, também foi observada em um estudo realizado por Paine et al. (1985).

Infelizmente, a Faculdade de Medicina não está cumprindo seu papel de alertar os estudantes sobre os malefícios do tabagismo, uma vez que observou-se um aumento na prevalência de fumantes nos últimos anos do curso médico. Resultado semelhante foi também encontrado em um estudo realizado com os estudantes da Faculdade de Ciências Médicas em São Paulo (Rosemberg \& Peron, 1990). Este fato não pode ser explicado simplesmente pela maior faixa etária nestas séries. Parece-nos que, se as faculdades de medicina estivessem cumprindo as re- comendações da $23^{\mathrm{a}}$ e da $24^{\mathrm{a}}$ Assembléia Mundial de Saúde, os estudantes estariam mais bem informados acerca dos perigos do tabaco à saúde (OMS, 1974) e o tabagismo certamente apresentaria um perfil epidemiológico diferente em nossas universidades.

Tem sido proposto que as escolas de medicina ofereçam ao seus alunos programas educacionais sobre os malefícios do fumo (Glanz et al., 1991), o que não tem sido feito pela maioria de nossas escolas médicas.

Sendo o controle do tabagismo uma das principais áreas da Medicina Preventiva, salientamos a necessidade de uma maior atenção, por parte dos órgãos competentes das faculdades de medicina, para que os estudantes recebam efetivamente todo o conhecimento necessário referente aos problemas causados por tal hábito.

Infelizmente, não apenas o conhecimento dos malefícios do fumo sobre a saúde deveria ser abordado nos programas de controle do tabagismo, e, sim, uma abordagem multidisciplinar envolvendo a dependência física e psíquica para que efetivamente mudanças comportamentais possam ter sucesso.

\section{RESUMO}

MENEZES, A. M. B.; HORTA, B. L.; ROSA, S.; OLIVEIRA, F. K. \& BONNANN, $M$. Hábito de Fumar entre Estudantes de Medicina da Universidade Federal de Pelotas, Brasil: Comparação entre as Prevalências de 1986 e 1991. Cad. Saúde Públ., Rio de Janeiro, 10 (2): 164-170, abr/jun, 1994. O presente estudo teve como objetivo investigar a tendência temporal no hábito de fumar entre os estudantes de Medicina da Universidade Federal de Pelotas, através de entrevistas com todos os alunos matriculados nos cinco primeiros anos do curso, em 1986 e 1991. Os percentuais de perdas foram de $4 \%$ e $2 \%$, respectivamente. Houve uma diminuição na prevalência de fumantes de $21,6 \%$, em 1986 , para 14,6\%, em 1991. Os percentuais de ex-fumantes foram de 7,3\% e 10,9\%, respectivamente. Não foi encontrada uma relação entre sexo e hábito de fumar assim como não houve associação entre o tabagismo de pais e filhos. Em 
1991, o percentual de fumantes foi maior nas duas últimas séries do curso; a sintomatologia respiratória mostrou-se associada significativamente com o hábito de fumar. Concluindo, o presente estudo mostra que o tabagismo está se tornando menos comum entre os estudantes de Medicina da Faculdade de Medicina de Pelotas.

Palavras-Chave: Epidemiologia; Tabagismo; Sintomatologia Respiratória

\section{REFERÊNCIAS BIBLIOGRÁFICAS}

DOLL, R., 1986. Tobacco: an overview of health effects. In: Tobacco: a major International Health Hazard (D. Zaridze \& R. Peto, eds.), pp. 11-22, Lyon: International Agency for research on Cancer.

IARC (International Agency for Research on cancer), 1986. IARC Monographs on Evaluation of the Carcinogenic Risk of Chemicals to Humans: Tobacco Smoking. Lion: International Agency for Research on Cancer. (IARC Scientific Publication 38).

GLANZ, K.; HEWITT, A. M. \& FIEL, S. B., 1991. Preventive pulmonary medical education. A review of its importance, status and challenge Chest, 100: 487-493.

LOKSCHIN, F. L. \& BARROS, F. C. 1983 Smoking or health: The Brazilian option. New York State Fournal of Medicine, 83: 13141316.

OMS Organización Mundial de la Salud), 1974. Consecuencias del tabaco para la salud. Genebra: OMS.(Série Informes Técnicos 568)
PAINE, P.; AMARAL, J. A. \& PEREIRA. M. G., 1985. Association between parental and student smoking in a Brazilian medical school. International fournal of Epidemiology, 14: 330-332.

POLLIN, W., 1982. Why People Smoke Cigarettes? Paper presented to the U.S. Congress, march 1982. Washington, D.C.: National Institute on Drug Abuse.

POLLIN, W. a\& RAVENHOLT, R. T., 1985. Tobacco addiction and tobacco mortality: Implications for death certification. Fournal of the American Medical Association, 252: 2849-2854.

RAVENHOLT, R. T., 1985. Tobacco's impact on twentieth-century U. S. Mortality patterns. American Fournal of Preventive Medicine, 1: 4-17. , 1990. Tobacco's global death march. Population and development Review, 16:213240.

RICHARDS, I. D. G. \& BAKER, M. R., 1988. Diseases of the Respiratory System. The Epidemiology and Prevention of Important Diseases. Edinburgh: Churchil Livingstone.

ROSEMBERG, J. \& PERON, S., 1990. Tabagismo entre estudantes da Faculdade de Ciências Médicas de Sorocaba. Tabagismo nos acadêmicos de medicina e nos médicos. Fornal de Pneumologia, 16: 13-22.

SANDE, J. L. M., ROSÓN, J. R. C.; ORCAJO, N. A. \& LLORIÁN, A. R., 1990. Estudio del tabaquismo en profesionales del medio hospitalario de Asturias. Revista Española de Cardiologia, 43: 219-226. 